\title{
Un modelo web para la asistencia en la toma de decisiones en la integración de las construcciones rurales mediante planificación espacial multi-criterio
}

\author{
A decision-supporting web model for integrating rural buildings with multi- \\ criteria spatial planning
}

J. S. Jeong ${ }^{(*)}$, L. García-Moruno ${ }^{(*)}$, J. Hernández-Blanco ${ }^{(* *)}$

RESUMEN

Este trabajo presenta un modelo web ideado para asesorar en la toma de decisiones en la integración de construcciones turísticas rurales en su entorno, mediante el intercambio de información y colaboración entre los agentes implicados. Se expone el uso de metodologías espaciales para la selección de localizaciones adecuadas a partir de la evaluación de criterios múltiples (ECM) en un marco web. Se emplean cuatro criterios para la evaluación, que se muestran en un mapa final global de idoneidad, basado en cuatro mapas intermedios parciales según los criterios de evaluación. Como resultado se utilizan tres niveles de información: página de información general, página para la toma de decisión espacial multi-criterio y una página con mapas de intercambio de conocimiento. El modelo propuesto, aplicado al caso práctico de 'Hervás', pretende conseguir una discusión esclarecedora entre distintas percepciones de integración de construcciones en su entorno, considerando diferentes alternativas de decisión y posibles contribuciones.

Palabras clave: Planificación colaborativa espacial; integración de construcciones rurales; web; análisis multi-criterio; toma de decisiones.

\section{SUMMARY}

This paper presents an interoperable web-based model able to interchange information amongst different stakeholders and to use the information as a means of promoting collaborative activities to integrate rural tourism buildings into a landscape with a case study, Hervás (Spain). Preliminary results from a continuing research are explained with a spatial methodology for selecting the suitable locations of rural buildings, coupling multi-criteria evaluations (MCE) into a web framework. The aim of this methodology is to classify four evaluation criteria and then is to show a final composite suitability map based on the four intermediate maps. The model deals with three types of information: a general overview page; a multi-criteria spatial decision-supporting page; and, a knowledge sharing map page. Using the proposed model, the regional spatial planning is intended to discuss different perceptions of building integration with the surroundings from various decision alternatives and to elucidate this model's contribution.

Keywords: Collaborative spatial planning; rural building integration; web; multi-criteria analysis; decision-making.

(*) Centro Universitario de Mérida, Universidad de Extremadura. Mérida (España).

${ }^{(* *)}$ Centro Universitario de Plasencia, Universidad de Extremadura. Plasencia (España).

Persona de contacto/Corresponding author: jin@unex.es (J. S. Jeong).

Cómo citar este artículo/Citation: Jeong, J.S., García-Moruno, L., Hernández-Blanco, J. (2014). Un modelo web para la asistencia en la toma de decisiones en la integración de las construcciones rurales mediante planificación espacial multi-criterio. Informes de la Construcción, 66(533): eoo4, doi: http://dx.doi.org/10.3989/ic.13.001.

Licencia / License: Salvo indicación contraria, todos los contenidos de la edición electrónica de Informes de la Construcción se distribuyen bajo una licencia de uso y distribución Creative Commons Reconocimiento no Comercial 3.o. España (cc-by-nc). 


\section{INTRODUCCIÓN}

En las últimas décadas se ha producido una transformación, en muchos casos abrupta y discordante, entre las edificaciones rurales y su entorno (1) (2). Actualmente el turismo se considera como una herramienta de gran alcance para el desarrollo, estimulando el crecimiento económico, el aumento de divisas, la inversión a pequeña escala y el empleo local (3). Las políticas europeas de ordenación del paisaje establecen códigos para la construcción con el objetivo de proteger la identidad cultural y particularidades de las edificaciones y promover la calidad del paisaje (4). En muchos casos, el desarrollo del turismo conlleva una mayor protección del medio ambiente y el aumento de los fondos para la conservación (5). Sin embargo, la adecuada integración de las construcciones en su entorno, no es un factor que se considere habitualmente en la redacción de los proyectos de ingeniería y arquitectura (6) (7). Por lo tanto, es importante que los profesionales propicien una integración respetuosa y tengan presente el emplazamiento para equilibrar armónicamente las construcciones rurales con su entorno paisajístico, todo ello asociado con el turismo (8) (9).

En el sector turístico, el desarrollo sostenible ha sido considerado ampliamente, ya que satisface demandas atractivas para los turistas, protege el entorno y proporciona oportunidades para el crecimiento económico gracias a la coexistencia de un turismo de calidad y la protección del medio ambiente (10). La mayor preocupación existente hacia los aspectos ecológicos y del patrimonio cultural está motivando mejoras en los entornos locales, incrementándose así su atractivo visual, sus valores estéticos y recreativos (11). Coincidiendo con un fuerte desarrollo urbanístico en Extremadura a finales del siglo $\mathrm{XX}$, se ha incrementado de forma notable el número de edificaciones en zonas rurales. La incorporación en el entorno rural de estas nuevas edificaciones ha supuesto un impacto en la propia forma de las construcciones, así como en sus relaciones con el entorno rural actual (6) (12) (13). La planificación regional actual no ha sido efectiva a la hora de considerar la evolución de los entornos rurales. En concreto no se han considerado ciertos criterios que podrían haber mitigado el impacto que han supuesto determinadas construcciones en su entorno rural (8) (9) (14). Con buenas prácticas incluso se podría haber enriquecido el entorno.

La modelización espacial, que proporciona la utilización de sistemas de información geográfica (SIG), permite analizar gran cantidad de datos espaciales mostrando mapas basados en datos georreferenciados en las informaciones económicas, sociales, culturales y ecológicas desarrolladas por la sociedad (15) (16). Además, el SIG proporciona herramientas para estudiar minuciosamente las diferentes localizaciones teniendo en cuenta limitaciones en la ordenación del territorio, oportunidades o características visuales que tengan en cuenta la escena paisajística general (17) (18) (19) (20). A partir de los modelos generados, los responsables de la planificación pueden determinar el estado actual y obtener una idea de las condiciones futuras. Incluso se podrán considerar las posibles consecuencias de los planes de ordenación del territorio y las políticas que puedan estar considerando (21). La evaluación de criterios múltiples (ECM) es una herramienta que pretende ser útil para la ordenación del territorio y facilitar a los responsables la exploración y resolución de problemas con gran cantidad de variables y datos (22). Los problemas de la ordenación del territorio suelen incorporar gran número de partes interesadas con diferentes antecedentes, inquietudes e interpretaciones de las dificultades (23). Por este motivo, el trabajo colaborativo puede ser una opción adecuada para aunar las aportaciones individuales a los problemas asociados con la ordenación del territorio y tomar decisiones que satisfagan sino a la totalidad, al menos a la mayoría de los afectados (24). En la última década, se han realizado numerosos esfuerzos para desarrollar una herramienta con estas características que integre y sea capaz de tratar «dentro de un marco único» tanto la vertiente analítica como la comunicativa de los procesos de diseño y ordenación del territorio (24) (25). La definición de este marco único posee importancia crítica. Se ha demostrado que la utilización de internet proporciona un mecanismo fundamental para garantizar que las partes interesadas tengan la oportunidad de participar en el proceso del diseño y ordenación del territorio en un proceso de colaboración asincrónico y distributivo (26).

El objetivo del presente trabajo es, por tanto, analizar y examinar cómo la investigación que se propone puede contribuir en los procesos de toma de decisiones entre las partes interesadas para la integración de construcciones turísticas rurales y su entorno, mostrando la funcionalidad de esta propuesta a través del estudio en Hervás, España. Este trabajo se centra en el diseño de una aplicación web basada en sistemas ECM combinados con la ponderación aditiva simple (PAS), con la que se podrá identificar y formular criterios apropiados y modelos espaciales para la correcta integración en la ordenación del territorio, con el objetivo principal de resaltar las interrelaciones entre las construcciones rurales y su entorno. La aplicación desarrollada en este estudio constituye una nueva alternativa que intenta facilitar los procesos de toma de decisiones, cuantificando la percepción de los usuarios, archivando los mapas de conocimiento que podrán ser compartidos y reutilizados posteriormente y verificando el impacto económico de las acciones tomadas sobre el turismo. Así, esta aplicación podría ser considerada como un vía de colaboración y comunicación entre los usuarios con intereses específicos o particulares en la ordenación del territorio en entornos rurales. La metodología propuesta se ha aplicado a un caso de estudio, mostrándose y discutiéndose los resultados en este trabajo.

\section{MATERIALES Y MÉTODO}

\subsection{Descripción del área de estudio}

Se ha establecido Hervás como área de estudio. Esta población está localizada en la comarca del Valle del Ambroz al norte de la provincia de Cáceres (Extremadura, España), como se indica en la Figura 1. El área de estudio tiene una superficie total de $60 \mathrm{~km}^{2}$. Por su mayor número de habitantes, Hervás es el centro administrativo y comercial de la comarca del Valle del Ambroz. En esta comarca, el terreno tiene un uso multifuncional mediante un sistema agro-silvestre y de pastoreo centrado en la Dehesa. Constituye un paisaje cultural específico, en el que predominan los bosques caducifolios de castaños, que son la base de un importante conjunto de empresas. Además, este área posee un importante valor biológico, escénico y recreacional, con un gran número de ríos y humedales idóneos para el destino turístico en periodos estivales.

En el comienzo de la década de los noventa, se produjo un importante cambio en este área hacia un desarrollo rural sostenible, como consecuencia de diferentes iniciativas europeas en Extremadura (Proyectos LEADER y PRODER) 


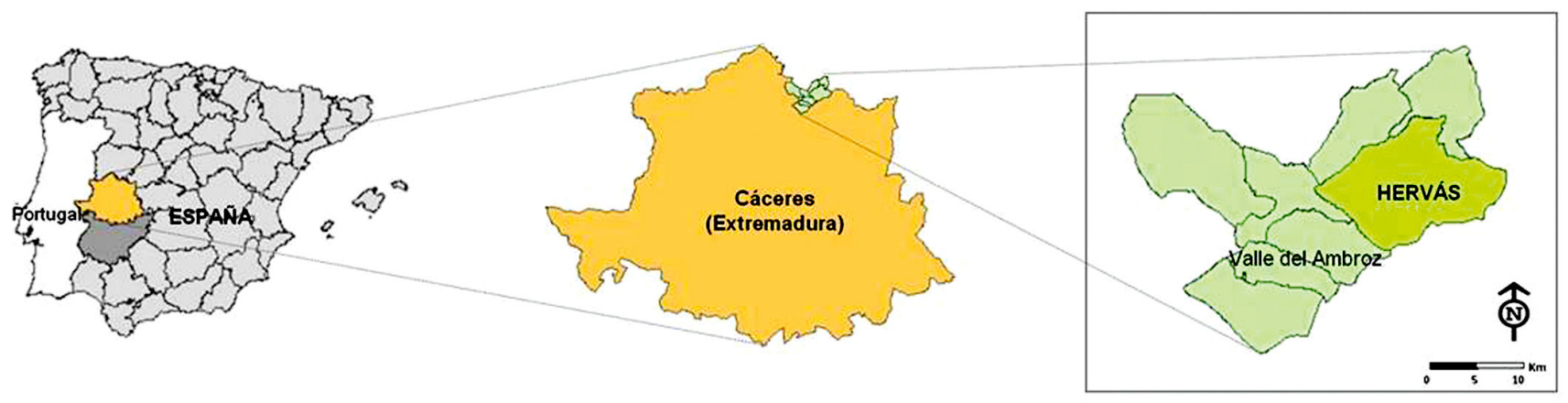

Figura 1. Mapa de situación del área de estudio Hervás.

(27). Durante las últimas décadas ha crecido el desarrollo de edificaciones en entornos rurales, muchas de ellas segundas residencias con fines vacacionales. También se ha producido un importante desarrollo de las actividades relacionadas con el turismo rural, lo que ha conllevado un incremento en la construcción de hoteles y casas rurales (28). Este proceso ha provocado un impacto, algunos estudios ya han resaltado que el continuo desarrollo en entornos urbanos y rurales ha causado importantes cambios en el uso de territorio, lo que se refleja en la pérdida de los entornos tradicionales (28) (29). En un corto período de tiempo se han realizado grandes cambios en la utilización del suelo como consecuencia del enorme desarrollo turístico y urbanístico. La respuesta a la situación actual (LESOTEX, Ley 15/2001 del suelo y ordenación territorial de Extremadura) está vinculada con la ordenación regional del territorio mediante planes, programas y diferentes acciones incluyendo las repercusiones territoriales. Sin embargo no proporciona una respuesta apropiada al problema real planteado (30). De hecho, los cambios que se producen con el desarrollo rural son más rápidos que la capacidad que tiene el legislador para comprenderlos (31). Las actuales políticas fallidas e instrumentos de planeación necesitan modificaciones y/o nuevas alternativas que deben ser desarrolladas e implementadas. Además, el proceso de planificación debe ser sometido a debate público para obtener sugerencias, alternativas, objeciones u otros puntos de vista mediante la colaboración con otras asociaciones y particulares.

\subsection{Evaluación de idoneidad del área}

Con objeto de determinar la localización más apropiada para la integración en su entorno de edificaciones rurales con fines turísticos se han aplicado diversos métodos, como la superposición e indexado, considerando diferentes parámetros a través del uso de herramientas de análisis espacial tales como SIG complementado con ECM. Los criterios de evaluación utilizados en esta investigación han sido clasificados en cuatro categorías principales: criterios físicos, ambientales, sociales y económicos. Todos han sido implementados en un proceso computacional y seleccionados a partir de la consulta de literatura relevante, políticas regionales y las directrices de la Unión Europea.

Se han considerado un amplio número de criterios de evaluación que influyen en la integración de las edificaciones turísticas rurales y se han tenido en cuenta diferentes etapas de evaluación que se han identificado con los cuatro criterios principales: físicos, medioambientales, sociales y económicos. Cada uno de estos criterios principales lleva asociado diferentes sub-criterios. En concreto, se han considerado los 16 subcriterios en el proceso computacional como se indica en la Ta- bla 1: (a) aspecto, (b) elevación, (c) pendiente, (d) visibilidad, (e) la presencia de un ecosistema sensible de acuerdo con la regulación de la Comisión Europea para la naturaleza y la biodiversidad (NATURA 2000) (32), (f) presencia de fuentes agua, (g) presencia de agua superficial, (h) tipo de vegetación, (i) proximidad de áreas residenciales, (j) densidad de población, (k) proximidad a áreas culturales, (l) accesibilidad, (m) proximidad a núcleos urbanos, (n) proximidad a zonas turísticas, (o) proximidad a zonas agrícolas y (p) utilización del territorio y tipo de cubierta. De modo que el criterio principal «físico» incluye los sub-criterios del (a) al (d), el criterio «medioambiental» incluye los sub-criterios del (e) al (h); el tercer criterio «social» incluye los sub-criterios del (i) al (l) y finalmente, el criterio «económico» incluye los criterios del (m) al (p).

La estructura jerárquica del proceso de decisión consta de cuatro niveles. El primer nivel muestra el objetivo principal: la idoneidad de la edificación turística rural. El segundo nivel representa los criterios que apoyan al objetivo principal. El tercer nivel corresponde los sub-criterios de cada uno de los criterios principales. Por último, el cuarto nivel muestra los atributos espaciales de cada sub-criterio.

La idoneidad de la localización de la construcción turística rural, considerando su entorno, es determinada mediante la combinación de los criterios de evaluación indicados en la metodología propuesta. De este modo, comenzando con el proceso analítico jerárquico (PAJ), se extraen los pesos de importancia relativa con la matriz de comparación por pares (MCP) y los valores de puntuación mediante la evaluación y juicio de la situación actual de los indicadores de cada criterio. En pocas ocasiones se obtendrá la MCP totalmente coherente (33) (34) (35). La estructura que se establezca en la MCP por parte de los tomadores de decisiones dependerá de la importancia con la que perciban cada uno de los criterios, para ello se establece una escala de puntuación como la indicada en la Tabla 2 (36). En este estudio, se ha empleado una escala de nueve puntos que obedece a los siguientes atributos, a $\ddot{i}=1$ $\mathrm{y}$ a $i j=1 /$ aji. El método PAJ incluye un índice denominado Razón de Consistencia (RC) que indica la coherencia global de la MCP (37) (38). Según Saaty (34), la RC debe tener un valor de menos del 10\%, para considerar que una matriz sea consistente. El peso calculado de prioridad de todos los criterios y sub-criterios «una vez establecida la MCP por parte de los autores» se presenta en la Figura 2. Los parámetros PAJ indican las asignaciones de los pesos de importancia relativa final considerados para un primer acercamiento al problema.

Finalmente, la aplicación de la PAS se utiliza con una escala de 9 puntos para extraer la información de los cálculos del índice 
Tabla 1.

Los cuatros criterios principales y su desglose en sub-criterios.

\begin{tabular}{|c|c|c|}
\hline Criterio & Sub-criterio & Descripción del sub-criterio \\
\hline \multirow{4}{*}{ Físicos } & Aspecto & $\begin{array}{l}\text { Muestra las áreas con buena orientación, desde un punto de vista estético. Constituye } \\
\text { un parámetro básico junto con la elevación y la pendiente. }\end{array}$ \\
\hline & Elevación & $\begin{array}{l}\text { Muestra las áreas de elevación expresada en metros, parámetro básico de la superficie } \\
\text { del suelo y de los procesos atmosféricos con efecto en los atributos derivados } \\
\text { medioambientales. }\end{array}$ \\
\hline & Pendiente & $\begin{array}{l}\text { Muestra las áreas de pendiente expresada en grados, parámetro con efecto en los } \\
\text { atributos derivados medioambientales y en el aspecto del paisaje de acuerdo } \\
\text { a la forma y flujo de la superficie del terreno. }\end{array}$ \\
\hline & Visibilidad & $\begin{array}{l}\text { Muestra las áreas destinadas a la protección estética utilizando las distancias radiales } \\
\text { y visibles desde puntos de acceso a la localización, tales como carreteras } \\
\text { (autopistas y carreteras locales) y los ferrocarriles. }\end{array}$ \\
\hline \multirow{4}{*}{ Medioambientales } & Ecosistema sensible & $\begin{array}{l}\text { Muestra las áreas calculadas utilizando funciones de distancia Euclidianas, la distancia } \\
\text { radial desde el ecosistema sensible de acuerdo con NATURA } 2000 .\end{array}$ \\
\hline & Fuentes agua & $\begin{array}{l}\text { Muestra las áreas calculadas utilizando funciones de distancia Euclidianas, distancia } \\
\text { en línea recta a masas de aguas, manantiales y/o pozos. }\end{array}$ \\
\hline & Agua superficial & $\begin{array}{l}\text { Muestra las áreas calculadas utilizando funciones de distancia radial de fuentes de } \\
\text { aguas superficiales, lagos y/o ríos con flujos continuos de agua. }\end{array}$ \\
\hline & Tipo de vegetación & $\begin{array}{l}\text { Muestra las áreas que incluyen una evaluación basada en la singularidad ecológica } \\
\text { de vegetación y las áreas deforestadas. Se muestra la extensión natural de estas } \\
\text { formaciones naturales. }\end{array}$ \\
\hline \multirow{4}{*}{ Sociales } & Áreas residenciales & $\begin{array}{l}\text { Muestra las áreas calculadas utilizando funciones de distancia Euclidianas, la distancia } \\
\text { radial desde ciudades y pueblos que representan actividad humana. }\end{array}$ \\
\hline & Densidad de población & $\begin{array}{l}\text { Muestra las áreas de las zonas de influencia alrededor de una ciudad, pueblo } \\
\text { o asentamiento humano asociado con la distancia económica basado en la información } \\
\text { facilitada por el instituto nacional de estadística (INE). }\end{array}$ \\
\hline & Áreas culturales & $\begin{array}{l}\text { Muestra las áreas que poseen aspectos culturales del área de estudio, que presentan } \\
\text { zonas para la protección de herencias culturales de acuerdo con las restricciones legales. }\end{array}$ \\
\hline & Accesibilidad & $\begin{array}{l}\text { Muestra las áreas calculadas mediante funciones de distancia radial, la distancia } \\
\text { directa desde las estructuras de los puntos de acceso, tales como autopistas, } \\
\text { carreteras y vías de tren. }\end{array}$ \\
\hline \multirow{4}{*}{ Económicos } & Núcleos urbanos & $\begin{array}{l}\text { Muestra las áreas calculadas usando funciones de distancia Euclidianas, la distancia } \\
\text { directa desde las zonas urbanas basada en el uso del terreno y el tipo de cubierta. }\end{array}$ \\
\hline & Zonas turísticas & $\begin{array}{l}\text { Muestra las áreas en las que se mezclan zonas culturales y urbanas, incluyendo zonas } \\
\text { de interés arqueológico, mediante el uso de funciones de distancia Euclidianas. }\end{array}$ \\
\hline & Zonas agrícolas & $\begin{array}{l}\text { Muestra las áreas con explotaciones agrarias en proceso de cambio de uso de terreno, } \\
\text { con la distancia mínima de protección según las restricciones legales. }\end{array}$ \\
\hline & Utilización del territorio & $\begin{array}{l}\text { Muestra las áreas destinadas al desarrollo económico que incluya el uso de la tierra } \\
\text { y los tipos de cobertura basado en modelo de elevación digital (LESOTEX). }\end{array}$ \\
\hline
\end{tabular}

de aptitud. Así se calculan los valores de clasificación finales en problemas con criterios múltiples basándose en la escala de clasificación utilizada en el presente trabajo para el índice de idoneidad comprendido entre o y 10, el cual establece la zona menos o más apropiada según se indica en la Ecuación [1] (39) (40). Cada indicador muestra una puntuación, que puede ser comparada respecto al estado actual del indicador para alguna condición deseada. De esta forma, la puntuación 9 establece la condición de rendimiento excelente, la 7 establece un rendimiento bueno, la 5 establece un rendimiento aceptable, la 3 establece un rendimiento razonable y la puntuación 1 indicaría una condición de rendimiento desfavorable para cada condición deseada. Indicadores con valores intermedios, 2, 4, 6 y 8 se emplean para puntuar cada condición deseada (36).

$$
\text { [1] } V_{i}=\sum_{j=1}^{n} w_{j} v_{i j}
$$

donde $V_{i}$ es el índice de idoneidad del área $i ; w_{j}$ es el peso de importancia relativa para el criterio $j ; v_{i j}$ corresponde al valor de evaluación para el área $i$ bajo el criterio $j$; $n$ es el número total de criterios. Los criterios de evaluación se combinan en una rejilla que incluye a todas las puntuaciones para cada una de las rejillas por separado. Cada uno de los valores de los criterios de evaluación es combinado en una rejilla compleja en el campo del atributo apropiado.

\subsection{El modelo web implementado}

Para el caso de estudio seleccionado, el entorno web implementa la información con el servicio de información de internet (SII), habilitando a los participantes para la toma de decisiones. La aplicación web implementada, descrita en este trabajo, se propone como una solución para la mejor integración de las edificaciones rurales en su entorno, como una herramienta para apoyar la toma de decisiones, para evaluar la percepción de los usuarios que tienen sobre la integración de dichas edificaciones, para compartir y reutilizar los mapas personales de conocimiento entre los usuarios y como un índice para verificar el impacto económico de las acciones tomadas sobre el turismo. La aplicación consta básicamente de un área de información 


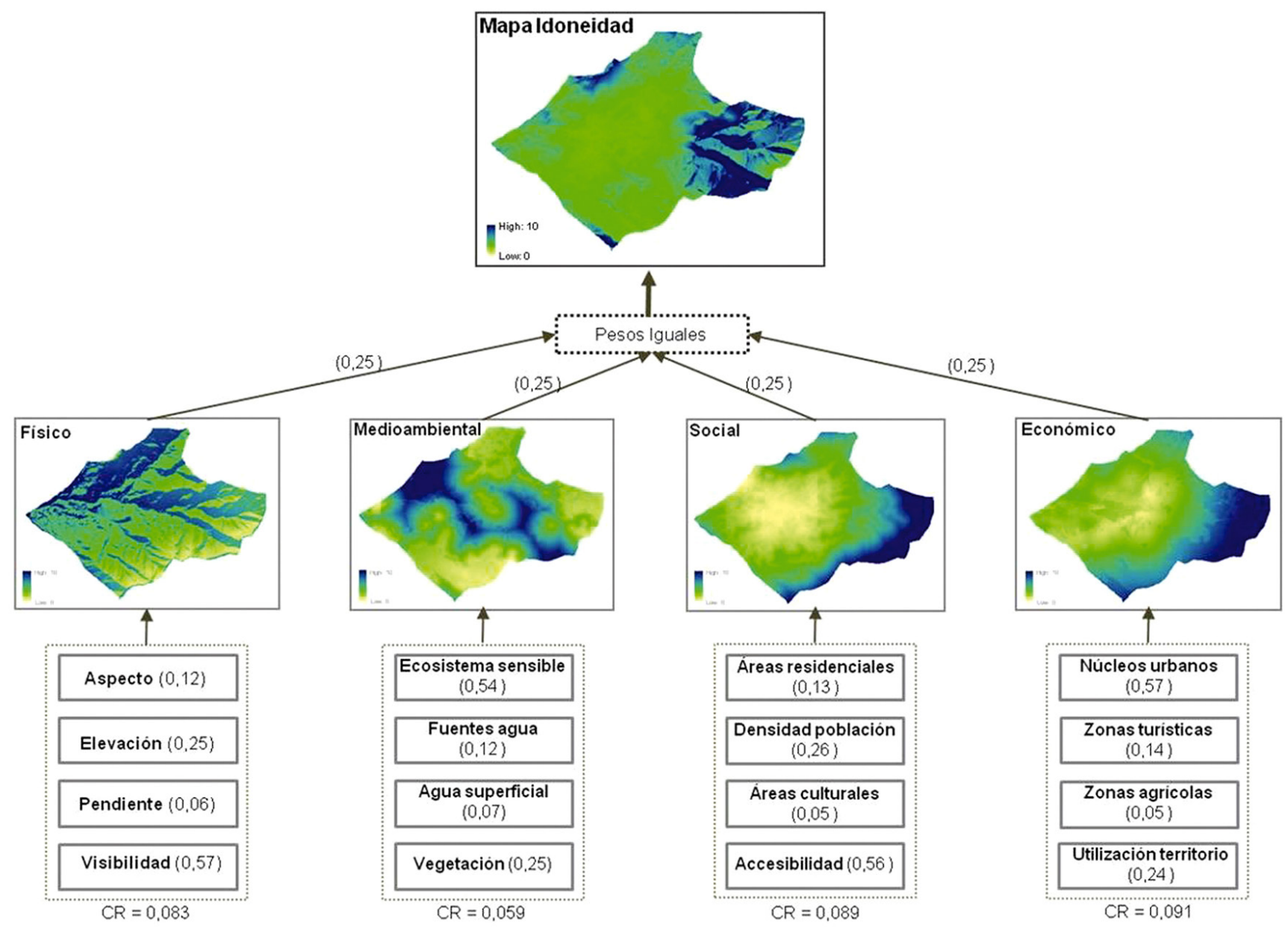

Figura 2. Organización jerárquica del proceso de decisión mediante los criterios de evaluación planteados.

Tabla 2. Matriz de comparación por pares para el cálculo numérico de los pesos de los criterios.

\begin{tabular}{|c|c|c|c|c|c|c|c|c|c|c|c|c|c|c|c|c|}
\hline \multicolumn{17}{|c|}{ Comparación por pares (Escala de valoración continua de 9 puntos) } \\
\hline \multicolumn{9}{|c|}{ Intensidad muy baja } & \multicolumn{8}{|c|}{ Intensidad muy alta } \\
\hline $1 / 9$ & $1 / 8$ & $1 / 7$ & $1 / 6$ & $1 / 5$ & $1 / 4$ & $1 / 3$ & $1 / 2$ & 1 & 2 & 3 & 4 & 5 & 6 & 7 & 8 & 9 \\
\hline \multicolumn{2}{|c|}{ Extrema } & \multicolumn{2}{|c|}{ Muy fuerte } & \multicolumn{2}{|c|}{ Fuerte } & \multicolumn{2}{|c|}{ Moderada } & \multicolumn{2}{|c|}{$\begin{array}{l}\text { Igual o } \\
\text { Equilibrada }\end{array}$} & \multicolumn{2}{|c|}{ Moderada } & Fuerte & \multicolumn{2}{|c|}{ Muy fuerte } & \multicolumn{2}{|c|}{ Extrema } \\
\hline
\end{tabular}

general, un área para la toma de decisión espacial multi-criterio y un área con mapas de intercambio de conocimiento, tal como se muestra en la Figura 3. Aunque el prototipo cuenta con estas tres etapas, puede considerarse como un entorno holístico y continuo con una barra de navegación superior y otros componentes visualmente coherentes con una página web.

El modelo web, denominado $e$-shift, dirige a los usuarios de forma consecutiva hacia el área de visión general, seguidamente a la zona de apoyo para la toma de decisión espacial multi-criterio y finalmente hacia el área de mapa de conocimiento. Los usuarios pueden navegar por este modelo de flujo de trabajo utilizando los botones de «siguiente» y «atrás» situados en la parte superior del área de trabajo principal. Además, $e$-shift cuenta con un menú de navegación en la parte superior, lo que permite a los usuarios diseñar su propio flujo de trabajo a través del sistema. En primer lugar, la zona de visión general proporciona información introductoria, un manual de usuario, información de contacto y el formulario de registro a través del cual el usuario pude acceder de forma completa al sistema y simplificar el acceso a otros recursos. En segundo lugar, el sistema de soporte para la toma de decisión espacial multi-criterio ayuda al usuario a la selección de la localización de la edificación considerando las complejidades del proceso espacial como se ha mencionado anteriormente en la sección 2.2. Así, cada etapa tiene su propia función para documentar sus conocimientos a través de la transcripción de comentarios en la parte inferior del área de trabajo principal. Se debe esperar que una única persona no se encuentre en posesión de todos los detalles y del conocimiento profundo para la integración total o parcial de una edificación. En tercer y último lugar, el área del mapa de conocimiento aglutina todas las partes de la aplicación incluyendo las transcripciones de los comentarios y el conocimiento tácito personal (41) y representa el recurso final para compartir y reutilizar la información generada por todos los usuarios. De este modo, los usuarios ven incrementada sus propias experiencias y su conocimiento a través del proceso de generación del mapa de conocimiento.

La aplicación web e-shift es funcional tanto con usuarios registrados en el sistema como aquellos que no lo estén. Todas las funciones del sistema están disponibles para aquellos 


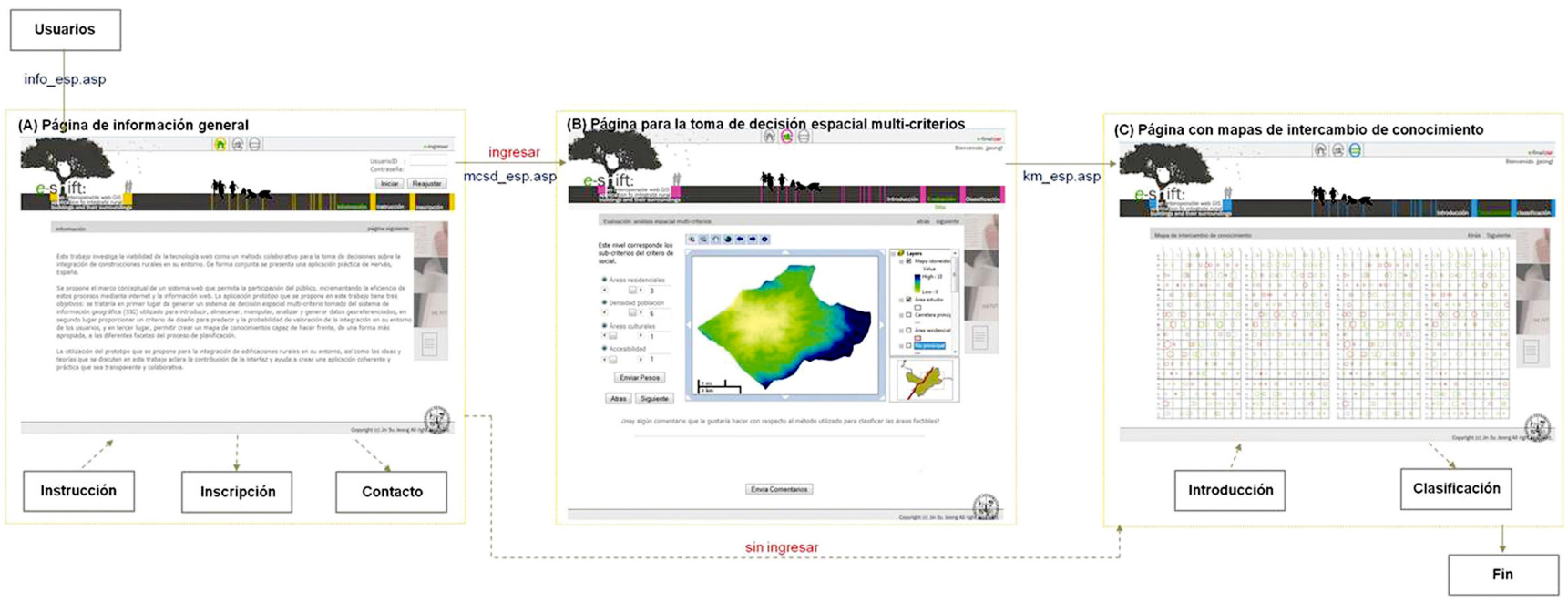

Figura 3. Despliegue del proceso del flujo de trabajo de $e$-shift.

usuarios que están debidamente autentificados. A aquellos usuarios que no se han registrado se les permite navegar por el sistema y leer toda aquella información que esté disponible, incluyendo las clasificaciones proporcionadas por otros usuarios, pero no se les permite participar activamente en el proceso de planificación espacial regional en su etapa segunda. Además, estos usuarios, no podrán clasificar los procesos de integración viables o contribuir a compartir y/o reutilizar los mapas de conocimiento. Para evitar este inconveniente, todas las páginas en el prototipo animan al usuario a registrarse. El sistema web diseñado detecta a los usuarios que regresan al sistema, cargando automáticamente la información enviada previamente, incluyendo los pesos de los criterios de decisión y las retroalimentaciones enviadas. Esta información puede ser editada en cualquier momento por parte del usuario.

\section{RESULTADOS Y DISCUSIÓN}

El modelo web implementado $e$-shift, permite la identificación del área más apropiada para la localización de la construcción rural con fines turísticos en Hervás. La utilización de la segunda etapa del modelo (el área de apoyo para la toma de decisión espacial multi-criterio), ilustra el posible escenario de agrupamiento utilizando cuatro criterios principales, los cuales se han combinado con dieciséis sub-criterios como en la etapa previa (Figura 4). Para obtener los posibles resultados alternativos, en primer lugar los usuarios exploran el área piloto para las construcciones rurales y otros componentes de integración del entorno y seguidamente expresan sus preferencias sobre dónde podría o no localizarse en función de los dieciséis sub-criterios. La asignación dada por este usuario sirve para establecer la importancia relativa del criterio de decisión que determina la asignación de los sitios viables para las categorías. Una vez evaluados todos los criterios de decisión, la última página muestra la clasificación de los resultados seleccionados para las localizaciones viables. A partir de este momento, los usuarios son más conscientes de la tarea en la que están involucrados y, sin duda, están en mejores condiciones para juzgar las relaciones de la zona de integración.

El mapa mostrado en la Figura 4a presenta una alternativa al escenario generado cuando el usuario ha asignado diferentes pesos a los criterios y sub-criterios establecidos. Esta alternativa aplica pesos iguales a los cuatro criterios. Además, este resultado muestra que la bondad del terreno se incrementa cuando el índice de idoneidad aumenta. Zonas con índices de idoneidad en el rango de $\mathrm{o}$ a 4 pueden considerarse, de forma general, como no idóneas para localizar una edificación rural con fines turísticos. Áreas con valores de índices de 9 a 10 pueden considerarse como las más apropiadas para la localización de la edificación rural con fines turísticos. En la Figura 4b, se muestra el resultado del porcentaje de área categorizada; en este caso la zona más apropiada es aquella con un 8,44\%. El resultado de agrupamiento también tiene interés para señalar los patrones espaciales diferenciadores generados mediante la asignación de pesos a los criterios y para indicar que la metodología presentada es capaz de revelar la zona más apropiada para la integración de las edificaciones rurales con fines turísticos en su entorno, así como proporcionar una escala inicial de zonas más apropiadas. De este modo, modelos integrados en un entorno web para la toma de decisión espacial multicriterio, como el modelo $e$-shift presentado en este trabajo, pueden ser de gran utilidad para tomar la decisión final.

Finalmente, en la última etapa, la clasificación conseguida se archiva en el mapa de conocimiento y se comparte y/o reutiliza para facilitar la comunicación entre los usuarios. El mapa de conocimiento es el último recurso de esta aplicación para la documentación, intercambio de información y reutilización por parte de los usuarios. Todos los comentarios de los usuarios se almacenan en una base de datos como registros personales del conocimiento tácito compartido. Esta información será accesible para todos los usuarios participantes en momentos posteriores y podrá utilizarse para intercambiar el conocimiento implícito generado anteriormente. Por ejemplo, los usuarios podrán leer las contribuciones aportadas con anterioridad y aprender sobre las perspectivas propuestas por otras personas sobre la idoneidad de una localización para albergar o construir elementos, así podrán modificar o revisar sus propias clasificaciones mediante el conocimiento de esta información.

\section{CONCLUSIONES}

En este trabajo se describe el diseño e implementación de un modelo web con metodología espacial, el cual permite afrontar y facilitar la integración de edificaciones rurales con fines turísticos en su entorno mediante la toma de decisión asincrónica. La percepción de integración de los usuarios, la elaboración de mapas de conocimiento y verificación económica 


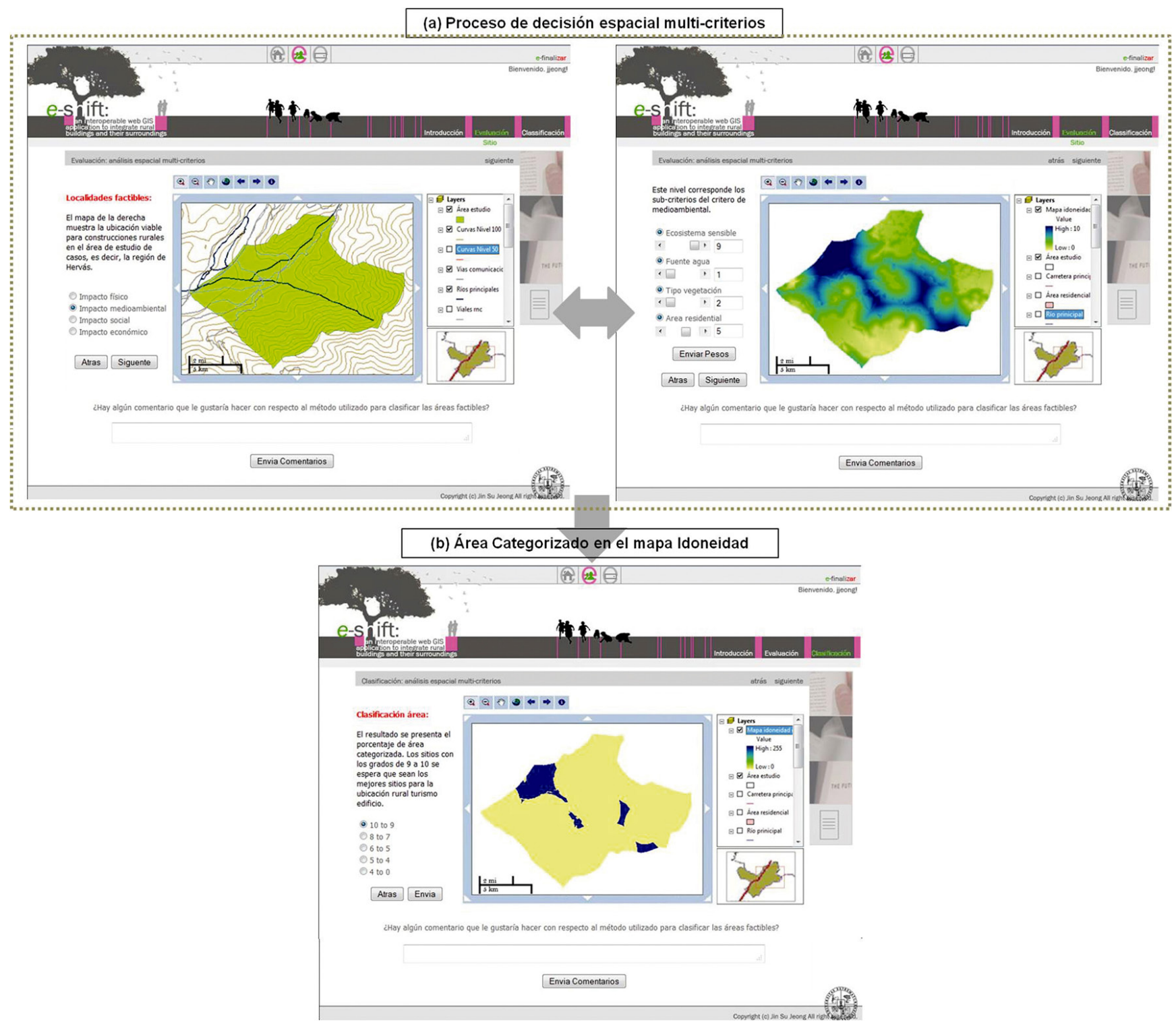

Figura 4. Selección de páginas web que muestran el proceso de toma de decisión espacial multi-criterio.

de los recursos turísticos. Para mostrar la aplicabilidad de este modelo, se ha desarrollado un caso práctico, utilizando el área de Hervás (Extremadura, España) como ejemplo. Particularmente, el caso de estudio ha presentado una aproximación de los procedimientos de agrupación mediante PAJ/PAS para generar un amplio rango de decisiones alternativas para abordar la problemática de la integración de edificaciones rurales con fines turísticos. A través del modelo web propuesto, los usuarios son capaces de aprender de forma interactiva e iterativa sobre la naturaleza del problema y además permite al usuario establecer sus preferencias para la solución deseada. El mapa de conocimiento generado, apoya y estimula el intercambio de opiniones y, por lo tanto, la discusión de intereses propios de cada usuario. Así, estos resultados preliminares muestran la flexibilidad del marco metodológico implementado en el modelo web, $e$-shift, más allá de la evaluación de todos los posibles criterios y sub-criterios.

Sin embargo, este modelo sólo será exitoso si todos los participantes en el proceso están dispuestos a poner en común sus conocimientos con objeto de incrementar el entendimiento y comprensión por todas las «partes» trabajando así hacia una visión común. Actualmente, el sistema se implementa como un concepto-prueba por parte de los autores. Será necesario llevar a cabo pruebas de idoneidad para la mejora del sistema. Se determinará si este sistema mejora el aprendizaje de los usuarios en todo el proceso y también se identificarán las instrucciones apropiadas para el uso del conocimiento. Durante las pruebas de evaluación de la aplicación prototipo, se tendrá en cuenta la usabilidad del software (42) para evaluar tanto la capacidad computacional como la interfaz gráfica del usuario (GUI). Una vez mejorada la aplicación prototipo, se llevarán a cabo un conjunto de encuestas y entrevistas para obtener información numérica sobre el rendimiento que los participantes han obtenido al utilizar el sistema, comprobando de este modo el beneficio potencial del modelo web propuesto.

\section{AGRADECIMIENTOS}

Los autores agradecen al Ministerio de Ciencia e Innovación (Proyecto BIA 2007-61166) por su financiación. Jin Su Jeong agradece a la Universidad de Extremadura por su contrato FPI del programa de Captación y Formación de Recursos Humanos de Excelencia en Investigación, Desarrollo e Innovación. 


\section{REFERENCIAS}

(1) de Vries, S., de Groot, M., Boers, J. (2012). Eyesores in sight: quantifying the impact of man-made elements on the scenic beauty of Dutch landscapes. Landscape and Urban Planning, 105(1-2): 118-127, doi: http://dx.doi.org/10.1016/j. landurbplan.2011.12.005

(2) Mennella, V. (1997). Qualità dell'a ambiente e sviluppo delle aree rurali. Genio Rurale, Edagricole, Perugia.

(3) De Kadt, E. (1979). Tourism: passport to development?. Oxford University Press, Oxford.

(4) Council of the European Union. (2001). Council resolution on architectural quality in urban and rural environments (2001/C 73/04). Official Journal of the European Communities, C73, 6/3/2001.

(5) Pigram, J. (1980). Environmental implications of tourism development. Annals of Tourism Research, 7(4): 554-583, doi: http://dx.doi.org/10.1016/0160-7383(80)90049-3.

(6) Jeong, J.S., García-Moruno, L., Hernández-Blanco, J. (2013). A site planning approach for rural buildings into a landscape using a spatial multi-criteria decision analysis methodology. Land Use Policy, 32: 108-118, doi: http://dx.doi. org/10.1016/j.landusepol.2012.09.018.

(7) Tassinari, P., Torreggiani, D., Paolinelli, G., Benni, S. (2007). Rural buildings and their integration in landscape management. Agricultural Engineering International: the CIGR Ejournal, vol. 9, Manuscript LW 07020.

(8) Bell, S. (1995). Elements of visual design in the landscape. E\&FN Spon, London.

(9) Tandy, C. (1979). Industria y paisaje. Ed Leonard Hill Books, Madrid.

(10) Eagles, P.F.J., McCool, S.F., Haynes, C.F. (2002). Sustainable tourism in protected areas: guidelines for planning and management. International Union for the Conservation of Nature, Gland, Switzerland.

(11) Zhang, H., Lei, S.L. (2012). A structural model of residents' intention to participate in ecotourism: the case of a wetland community. Tourism Management, 33(4): 916-925, doi: http://dx.doi.org/10.1016/j.tourman.2011.09.012.

(12) Dwyer, J.F., Childs, G.M. (2004). Movement of people across the landscape; a blurring of distinctions between areas, interests, and issues affecting natural resource management. Landscape and Urban Planning, 69(2-3): 153-164, doi: http://dx.doi.org/10.1016/j.landurbplan.2003.09.004.

(13) Van der Wulp, N.Y. (2009). Verrommeling van het landschap, de rol van storende elementen. Landschap, 26(3): 132144.

(14) García, L., Hernández, J., Ayuga, F. (2006). Analysis of the materials and exterior texture of agro-industrial buildings: a photo-analytical approach to landscape integration. Landscape and Urban Planning, 74(2): 110-124, doi: http://dx.doi. org/10.1016/j.landurbplan.2004.10.007.

(15) Böhme, K., Schön, P. (2006). From Leipzig to Leipzig: territorial research delivers evidence for the new territorial agenda of the European Union. disP, 165(2): 61-70.

(16) Hermann, S., Osinski, E. (1999). Planning sustainable land use in rural areas at different spatial levels using GIS and modelling tools. Landscape and Urban Planning, 46(1-3): 93-101, doi: http://dx.doi.org/10.1016/So169-2046(99)oo050-X.

(17) Domingo-Santos, J.M., Fernández de Villarán, R., Rapp-Arrarás, I., Corral-Pazos de Provens, E. (2011). The visual exposure in forest and rural landscapes: an algorithm and a GIS tool. Landscape and Urban Planning, 101(1): 52-58, doi: http://dx.doi.org/10.1016/j.landurbplan.2010.11.018.

(18) Hernández, J., García, L., Ayuga, F. (2004). Integration methodologies for visual impact assessment of rural building by geographic information system. Biosystems Engineering, 88(2): 255-263, doi: http://dx.doi.org/10.1016/j.biosystemseng.2004.02.008.

(19) García-Moruno, L., Hernández-Blanco, J., Ayuga-Téllez, F., García-Navarro, J. (1998). Localización de las construcciones en el ámbito rural ante la protección del paisaje. Informes de la Construcción, 50(458): 61-70, doi:http://dx.doi. org/10.3989/ic.1998.v50.i458.879.

(20) Tassinari, P., Torreggiani, D. (2006). Location planning: a methodological approach for agro-industrial buildings in rural territory. Transactions of the ASAE, 49(2): 505-516, doi: http://dx.doi.org/10.13031/2013.20405.

(21) Blaschke, T. (2006). The role of the spatial dimension within the framework of sustainable landscapes and natural capital. Landscape and Urban Planning, 75(3-4): 198-226, doi: http://dx.doi.org/10.1016/j.landurbplan.2005.02.013.

(22) Malczewski, J. (1999). GIS and multicriteria decision analysis. John Wiley \& Sons, New York.

(23) Fountas, S., Wulfsohn, D., Blackmore, B.S., Jacobsen, H.L., Pederson, S.M. (2006). A model of decision-making and information flows for information-intensive agriculture. Agricultural Systems, 87(2):192-210, doi: http://dx.doi. org/10.1016/j.agsy.2004.12.003.

(24) Jankowski, P., Nyerges, T., Smith, A., Moore, T.J., Horvath, E. (1997). Spatial group choice: a SDSS tool for collaborative spatial decision-making. International Journal of Geographical Information Systems, 11(6): 577-602, doi: http:// dx.doi.org/10.1080/136588197242202.

(25) Voss, A., Denisovich, I., Gatalsky, P., Gavouchidis, K., Klotz, A., Roeder, S., Voss, H. (2004). Evolution of a participatory GIS. Computers, Environment and Urban Systems, 28(6): 635-636, doi: http://dx.doi.org/10.1016/j.compenvurbsys.2003.12.003.

(26) Voinov, A., Bousquet. F. (2010). Modeling with stakeholders. Environmental Modelling \& Software, 25(11): 1268-1281, doi: http://dx.doi.org/10.1016/j.envsoft.2010.03.007.

(27) Nieto-Masot, A. (2011). Las políticas europeas LEADER y PRODER en Extremadura (España). El desarrollo rural en Extremadura: las políticas europeas y el impacto de los programas LEADER y PRODER. Mérida. Editorial Académica Española.

(28) Jaraíz, F.J., Mora, J., Gutiérrez, J.A., Jeong, J.S. (2013). Comparison of regional planning strategies: countywide general plans in USA and territorial plans in Spain. Land Use Policy, 30(1): 758-773, doi: http://dx.doi.org/10.1016/j.landusepol.2012.06.001. 
(29) Tassinari, P., Carfagna, E., Benni, S., Torreggiani, D. (2008). Wide-area spatial analysis: A first methodological contribution for the study of changes in the rural built environment. Biosystems Engineering, 100(3): 435-447, doi: http:// dx.doi.org/10.1016/j.biosystemseng.2008.04.004.

(30) LESOTEX. (2001). Guía práctica de aplicación de la Ley 15/2001 del suelo y ordenación territorial de Extremadura. http://sitex.juntaex.es/sias/Documentacion/guia_practica.pdf.

(31) Wascher, D., Múgica, M., Gulinck, H. (1999). Establishing targets to assess agricultural impacts on European landscapes. En Brouwer, F., Crabtree, B. (Eds.), Environmental indicators and agricultural policy (pp. 73-87). Wageningen.

(32) Natura 2000. European commission environment for nature \& biodiversity policy. http://ec.europa.eu/environment/ nature/natura20oo/index_en.htm.

(33) Armengou, J., Aguado, A., Ormazábal, G. (2012). Sistema integrado para toma de decisiones en el diseño de estructuras de hormigón. Informes de la Construcción, 64(527): 391-400, doi: http://dx.doi.org/10.3989/ic.11.019.

(34) Saaty, T.L. (1996). The analytic hierarchy process. New York. McGraw-Hill.

(35) Zeleny, M. (1982). Multiple criteria decision making. New York. McGraw-Hill.

(36) Balana, B.B., Mathijs, E., Muys, B. (2010). Assessing the sustainability of forest management: an application of multicriteria decision analysis to community forests in northern Ethiopia. Journal of Environmental Management, 91(6): 1294-1304, doi: http://dx.doi.org/10.1016/j.jenvman.2010.02.005.

(37) Golden, B., Harker, P., Wasil, E. (1989). The analytic hierarchy process: applications and studies. The Netherlands. Springer Verlag.

(38) Vargas, L., Zahedi, F. (1993). Analytic hierarchy process and its applications. Mathematical and Computer Modeling, 17: 4-5.

(39) Hwang, C.L., Yoon, K.L. (1981). Multiple attribute decision making: methods and applications. New York. SpringerVerlag.

(40) Yoon, K., Hwang, C.L. (1995). Multiple attribute decision making: an introduction. London. Sage Publications Inc.

(41) Polanyi, M. (1996). The tacit dimension. New York. Doubleday Broadway Publishing Group.

(42) Nielsen, J. (1994). Usability engineering. Boston. Academic Press Inc. 
